\title{
Measuring commitment to self-tracking: development of the C2ST scale
}

\author{
Elçin Hancı ${ }^{1} \mathbb{D}$ • Joyca Lacroix ${ }^{2}$ Peter A. M. Ruijten ${ }^{1} \cdot$ Antal Haans $^{1} \cdot$ Wijnand IJsselsteijn ${ }^{1}$
}

Received: 29 November 2019 / Accepted: 3 September 2020 / Published online: 17 September 2020

(C) The Author(s) 2020

\begin{abstract}
Self-tracking technologies bring a new set of experiences into our lives. Through sensors and ubiquitous measurements of bodily performance, a new form of automation experience shapes our understanding of our body and our behavior. While for many individuals self-tracking has an important role in their daily lives, a theoretical understanding of the level and behavioral manifestations of commitment to self-tracking is still missing. This paper introduces the concept of commitment to self-tracking and presents the development and first validation of a new 12-item behavior-based scale for its measurement, the Commitment to Self-Tracking $(C 2 S T)$ scale. Using online survey data from individuals wearing self-tracking technology $(N=300)$, we explore the underlying factor structure of the scale and determine its reliability and validity. An analysis of the survey data indicates that commitment to self-tracking positively correlates with autonomous motivation for tracking and negatively correlates with controlled motivation. The C2ST scale brings insights on how self-tracking technology, as a novel automation experience, is affecting users' everyday behaviors. Overall, by emphasizing the feasibility of defining commitment behaviorally, the paper concludes with implications for theory and practice and suggests directions for future research.
\end{abstract}

Keywords Self-tracking $\cdot$ Commitment $\cdot$ Quantified self $\cdot$ Measurement $\cdot$ Scale development

\section{Introduction}

For many individuals, self-tracking technologies have become an integral part of everyday automation experiences. Similar to other smart technologies, from voice-activated speakers to smart light bulbs, it is a form of automation that

Elçin Hancı

e.hanci@tue.ml

Joyca Lacroix

joyca.lacroix@philips.com

Peter A. M. Ruijten

p.a.m.ruijten@tue.nl

Antal Haans

a.haans@tue.nl

Wijnand IJsselsteijn

w.a.ijsselsteijn@tue.nl

1 Eindhoven University of Technology, PO Box 513, 5600 MB Eindhoven, The Netherlands

2 Philips Research, High Tech Campus 34, 5656 AE Eindhoven, The Netherlands affects our everyday life and enhances our capabilities. Uniquely, self-tracking technologies claim to facilitate a digital connection with our own bodies. The use of sensors and computing devices encompass a transformative capacity, converting one form of information (e.g., physical and physiological states) into forms of information that are subject to diagnosis and evaluation. Ultimately, the use of selftracking, by virtue of the automation experience it offers, transforms how we make sense of ourselves. It also tends to redefine the purpose of an activity - once we start measuring an activity, in a sense, it becomes a different activity altogether. The slogan of the Quantified Self (QS) movement, "Self-knowledge through numbers," highlights the potential gains in self-insights that might be acquired through objective self-assessment. Kevin Kelly, one of the founders of the Quantified Self community, refers to continuous self-tracking and feedback as "exo-senses" expanding the set of innate biological senses and the type of information we can acquire about the self. Combining self-tracking data with $\mathrm{AI}$, he argues, translates the collected data into meaningful insights, allowing for an immediate and intuitive way to "feel" the data in a very similar manner as the data that are coming in through our senses. 
Self-tracking devices are at the heart of the Quantified Self community with an emphasis on their potential role as behavior change technologies. These devices have been studied as effective tools in promoting self-awareness and facilitating behavior change. For example, the use of a pedometer significantly increases physical activity [4] and users that log their food intake more frequently with the help of their activity tracker lose more weight relative to low-frequency loggers [30]. Despite the widely acknowledged role as behavior change tools, self-tracking technologies have thus far largely been overlooked as automation experiences. Moving beyond the dedicated and tech-savvy QS community of selfquantifiers in the first decade of the twenty-first century, self-tracking is now as much a sociocultural phenomenon as it is a technological one. Viewing ourselves through a technological lens creates a novel understanding of who we are, for better or for worse. Self-tracking technology is thus becoming an increasingly potent tool in shaping the modern self-our sense of self-awareness, self-efficacy, and self-worth, our sense of our own bodies, our behaviors, our health, and social relations. In other words, it is a new form of everyday automation that deeply affects our experience of self.

The use of self-tracking brings along some implied norms that are embedded within the way feedback is delivered; what is deemed as "normal" vs. deviant for example is implicit in the mere fact that we use numbers, and the way in which numbers are presented, gauged, and compared. There appears to be very little room for personalization; users are nudged to think in terms of "what is normal in some absolute sense" rather than "what is normal for me" [43]. Moreover, tracking can be deemed paternalistic, imposing lifestyle changes and encouraging behavior change to people who may not benefit from such changes (e.g., [39]). There is an implied shaming of people who are not willing or able to live up to the body-centered ideals inherent in self-tracking [23]. More generally, self-tracking imposes an understanding of health that is part and parcel of a larger trend of biomedicalization as opposed to, for example, richer experiential, cultural, or historical notions of health. In his essay, Till [42] addresses the automation aspect of digital technology and draws the attention to the extent to which automation brings about the capability of transforming exercise into commodification. He claims that once commodified indirectly through marketing of products (e.g., running shoes that improve one's performance), being able to automatically log exercise (e.g., running) at a large-scale allows commodification of the exercise itself, what he refers to as "exercise as labor." In line with Till, other scholars too have criticized QS as a privacy risk, with one's "data double" living on servers of corporations, and being mined for patterns to be monetized as profitable propositions [3]. Perhaps the best known criticism is voiced through Shoshana Zuboff's work, who dubbed the term "surveillance capitalism" to refer to the information asymmetry related to data being shared with companies without us knowing how they use it, with whom the data is being shared, and how we are being targeted for commercial exploitation [46].

These criticisms tend to focus on how experience and interaction is turned into data, which is subsequently harvested and exploited by third parties, commercial, or otherwise. In a way, our focus in the current paper is on the mirror image of this process: how data about ourselves is influencing our own self-perceptions. By means of studying the affective, cognitive, social, and behavioral effects (that can be both negative and positive) of being confronted with numbers about oneself, we focus on the psychology of self-tracking. Finally, it's worthwhile noting that many of the above criticisms stem from principled positions (e.g., data ethics, feminist discourse, or discourse around body shaming), and not from empirical measurement (despite occasionally citing anecdotal evidence and examples). Psychology as the science of mind and behavior is less value-driven (i.e., what ought to be) and more datadriven (i.e., what is). Psychology tends to focus on empirical data when asserting claims about causal relationships.

To date, the field of personal informatics has only paid sparse attention to the psychological effects of self-tracking. We argue that investigating self-tracking technologies and their experiential effects through the lens of psychology brings a new understanding of the impact of these technologies. This is especially urgent given the fact that despite being a promising tool, self-tracking technologies face a challenge in that they struggle to deliver on what is promised, either implicitly or explicitly. That is, in order to engender sustained behavior change, users need to be engaged with self-tracking technologies and practices over a prolonged period of timelong enough to initiate and maintain new behavioral routines. However, keeping users psychologically engaged and motivated to use self-tracking appears to be a major challenge. For example, a survey revealed that over one-third of Americans who owned a self-tracking product abandoned it within 6 months [20]. Furthermore, findings of another study showed that tracking steps using a pedometer increased the number of steps of users but actually decreased their level of enjoyment of the activity [10]. These paradoxical findings suggest that more insight is required into the psychological mechanisms underlying the self-tracking practice, and its associated automation experiences. Correspondingly, today, more and more social scientists are turning their attention to the psychological and experiential effects of self-quantification. Having more tools to investigate the psychological impact of these technologies will help in improving our understanding of the underlying psychological processes, and may subsequently help in developing effective devices that support users' health and well-being in a sustained manner. Hence, the reasons for engaging in self-tracking and their behavioral and psychological manifestations become variables of interest when trying 
to gauge the psychological and behavioral impact of selftracking.

In this paper, we introduce a new construct of commitment to self-tracking and we present the development of a selfreport measure that is designed to assess people's commitment to self-tracking. Below, we will first introduce quantified self as a broad cultural phenomenon, part of the lived experience of many. Next, we will introduce commitment to self-tracking, and will compare this new construct to related constructs such as motivation. We then discuss the process of item generation, resulting in the Commitment to Self-Tracking (C2ST) scale and present a study through which we explore and validate the psychometric properties of our scale. We conclude with implications and possible applications of the scale for future research on self-tracking.

\subsection{Self-tracking practices}

Self-tracking technologies, such as fitness trackers, by means of providing ubiquitous and real-time monitoring, aim to increase self-awareness, thus prompting users towards making healthier lifestyle decisions [18]. Despite its prevalent usage in today's society, people vary in their engagement with their self-tracking devices and with the monitored feedback. While some users only track their number of steps simply because their smartphones have an embedded pedometer, others have bought dedicated devices to track their heart rate, glucose level, and sleep quality, for example. There also appears to be clear variation in the way people use their selftracking devices. While some users only occasionally check the numbers on their tracker, others are continuously engaged with the tracker and its various outputs, setting and adjusting goals, calculating progress, and sharing the numbers with other people using self-trackers and their broader online community. Self-quantifiers' behavioral engagement in relation to their tracking devices, as well as their self-tracking practices, appears to be an important factor in studying the psychological impact of self-tracking. Understanding the wide spectrum of behavioral and psychological manifestations of selftracking and how these relate to people's commitment to self-track will generate a deeper, more granular understanding of the phenomenon of self-tracking.

Many users acknowledge the benefits promised by selftracking technologies and are likely to include self-tracking in their attempts to adopt a healthier lifestyle [29]. People, however, differ in their motivations and intentions related to self-tracking. The Quantified Self community, for example, consists of a group of highly visible, dedicated, and techsavvy users who advocate a lifestyle with an emphasis on living by numbers. During QS show and tell meet-ups and QS conferences, they frequently share the various insights they have gained through their $N=1$ self-experimentation. Given their commitment and long-term adherence to self- tracking, various studies in the HCI domain have focused on the Quantified Self community, making this a very well-documented, even if somewhat extreme group of users. By using the publicly available video corpus of QS presentations, a number of studies have performed extensive qualitative research in understanding the different types of underlying motivations for self-tracking, resulting in a taxonomy of tracker profiles [5, 6, 21, 32, 33]. A closer look at these reasons and intentions for self-tracking can help in understanding people's commitment to engage in self-tracking. Recent work by Rooksby and colleagues, targeting the QS community, has distinguished between five different tracking styles. These are diagnostic tracking, documentary tracking, directive tracking, tracking to collect rewards, and fetished tracking [32]. Directive tracking concerns goal-driven tracking practices. A specific goal is set-for example to lose five kilograms of weight over six months - and after the goal is reached, the self-tracking is generally discontinued. In documentary tracking, people are intrinsically interested in documenting their activities; someone who has no heart condition but voluntarily keeps track of their heart data simply because they have a keen interest in understanding their body can be an example of this style of tracking. Diagnostic tracking, as opposed to documentary tracking, aims to find a link between one thing and another. For example, someone with sleep problems may start tracking daily alcohol and coffee intake, food habits, latenight media exposure, and other similar variables to uncover their relation to sleep quality. Collecting rewards is associated with another style of tracking where external rewards form the primary motivating factor for self-tracking. And lastly, fetished tracking involves users who have a pure interest in data, gadgets, and technology.

This taxonomy of user profiles demonstrates that reasons for self-tracking range from more autonomous and internally driven interests to controlled motivations that are primarily responsive to external incentives. Self-determination theory proposes that the type of motivation one experiences is an important determinant in predicting longevity and persistence of engaging in a behavior. While those who are motivated with autonomous reasons to self-track are more likely to carry on their practice independent from the presence of a reward and therefore exhibit more dedication, those whose primary motivation is determined by external drives, such as receiving rewards, are less likely to commit themselves to the self-tracking practice [7]. Therefore, it is important to note that commitment to selftracking is related to the internal states and drivers of a user, but not identical to it. We expect that intrinsically motivated users are more likely to commit to self-tracking than those who are in constant need of external motivations. Findings from related literature also indicate that commitment has been a relevant topic in the self-tracking domain but has been investigated rather implicitly. In the present paper, 
we aim to address commitment to self-tracking explicitly and conceptualize it as a separate concept from motivational drivers by being more clearly related to behavioral manifestations. We hypothesize that, in general, commitment to self-tracking correlates positively with autonomous motivation. Although one might expect commitment to correlate negatively with controlled motivation, this may not always be the case; for some particular user groups (e.g., those who track to collect rewards), the tracking may offer external incentives that become an end (as opposed to a means) in itself.

While acknowledging the close relation between commitment and motivation, it is noteworthy to highlight that these concepts stand as different constructs when it comes to understanding users' relationship with the self-tracking practice. Motivation, as a concept, does not necessarily require an action mainly because it describes an inner psychological state. This mere focus on internal states can be problematic as it is susceptible to the so-called intention-behavior gap, a phenomenon described as the discrepancy between a person's intention and taking the action necessary to operationalize the intended behavior [36]. Do users with different types of motivations also differ in terms of how they utilize the selftracking device? Unless these internal states are accompanied with a behavioral output, our ability to understand actual user behavior remains incomplete. For example, "I want to help/ inspire others" is one of the items developed by Gimpel and colleagues, with the purpose of measuring user's selfassociation motives for self-tracking [13]. It is known from literature that attitudes and intentions do not always equate to behavior [2]. Hence, although a user's response to this item may reflect the intended interest in being part of a self-tracking community, it is not yet clear whether this intention is translated into actual behavior.

Studies demonstrate that a user's understanding of selftracking devices can be more intricate than treating the device as a mere tool that delivers numbers; making sense of these quantified bodily insights seems to modify the social role of the device going beyond the role of a functional tool (e.g., by becoming a tutor or a friend), implying the emergence of social dynamics in the interaction [24]. We argue that investigating such a complex relationship only through the lens of motivational theories is insufficient in understanding such experiences fully and thoroughly. Behavioral commitment, in contrast to a person's motivational focus or inner drives, is defined by the actual state of action: when someone is committed, this commitment is expressed by an overt, measurable act. Thus, given that user's relation to self-tracking has implications both at individual and social levels that are manifested through one's behaviors, addressing one's commitment to self-track provides a more holistic understanding of a person's involvement into this selftracking culture.

\subsection{Commitment to self-tracking}

Commitment to self-tracking is conceptualized as a novel construct which provides a useful behavioral alternative to the aforementioned attitudinal approaches. Taking use patterns and motivations of QS members together with other studies on long-term self-tracking practices, we argue that commitment to self-tracking is a composite of several behavioral manifestations. In essence, these types of behaviors can be distinguished as follows: (1) immediate behaviors observed from a user's individual willingness to invest in self-tracking, (2) behaviors exhibited as a result of the direct interaction with the device, and (3) behaviors that manifest the social aspect of self-tracking. We will discuss each of these in more detail.

First, committed self-quantifiers experience their enthusiasm at a personal level. One of the founders of the QS community, Gary Wolf, tried to capture some of this sentiment: "most of us will admit that the utility of self-tracking in achieving some specified goal doesn't fully explain its fascination. There is a compulsion, a curiosity that seems to operate in advance of any particular use" [45]. Irrespective of the intended use, dedication to the technology is recognizable from the ways in which self-tracking is integrated into one's life. For example, by means of investing in the newest trackers (e.g., discarding older trackers for newer ones with more functionality), a dedicated self-tracker shows their commitment by putting extra effort in order to keep up-to-date with selftracking technology. Integrating self-tracking into one's life also includes providing means that yield a better integration; someone who is willing to spend more money in making the device more appealing for various occasions (e.g., purchasing an additional - not so sporty - wristband so that the fitness tracker can be worn when going out at night) hints at one's desire for uninterrupted self-tracking. Secondly, the level of commitment to self-tracking shapes the way in which a user interacts with the device. Interaction with the device concerns the ways it is being utilized (e.g., for diagnosis vs. for mere documentation) as well as how the monitored data is handled. Studies show that people who are dedicated to self-tracking differ in their approach towards analysis of self-data; not being satisfied with the monitored data at face value, highly committed users tend to take an extra step in reconstructing the data so that the interpretation gets enriched [5]. Thirdly, commitment takes place at a social level as much as it does at a personal level [17]. Despite the fact that self-tracking is essentially performed at an individual level, committed users share their data (e.g., at QS meet-ups, or in online communities), and develop a strong sense of belonging to a community to exchange experiences and learn from each other [22].

In light of the elements discussed above, commitment to self-tracking is defined as the collection of behaviors that indicate dedication to (i) self-trackers as devices, (ii) selftracking as a functional activity seeking to gather and interpret 
data on one's body and behavior, and (iii) self-tracking data as a part of one's projected social identity. We argue that commitment to self-tracking manifests itself in users' everyday activities and social interactions, simultaneously, which encompasses the time, money, and effort which users invest in self-tracking technologies, the variety of ways and contexts in which they use their self-trackers, and the extent to which they share (vs. keep to themselves) their self-tracking-related material, including the monitored data. By disentangling these behaviors, commitment to self-tracking aims at capturing the essence of the relationship a user has with self-tracking technology, and helps to operationalize the construct, thus enabling its efficient and meaningful characterization and comparisons across users. As can be inferred from the above definition, we regard commitment to self-tracking to reflect a single underlying factor, commitment, that is reflected in three different behavioral manifestations, as discussed.

A question that naturally follows from this is what kinds of behavior exactly are considered as committed behavior. In cases like job involvement, where norms are predefined with 9 to 5 working hours, it is rather straightforward to pinpoint what a committed behavior is; deviating from these norms and voluntarily spending extra hours from one's personal time can easily be a proxy for commitment to job involvement [44]. In self-tracking practices, on the other hand, there has been, to our knowledge, no systematic research that defines the behavioral routines of an average self-tracking user. Use patterns have been conventionally studied employing automatically registered data and demographic variables such as activity logs as a behavioral proxy of engagement [37]. In the context of digital behavior change, this traditional approach was recently challenged by Perski and colleagues who included both behavioral as well as experiential aspects of interaction as proxies for engagement [27]. However, scales measuring engagement with self-tracking technologies put an emphasis solely on the device that is being used. Commitment to self-tracking, as we define it here, adopts a more aggregated approach and highlights individual, behavioral as well as social aspects of self-tracking.

Overall, the relationship a user has with self-tracking technologies has been investigated using different approaches. Usage patterns have been studied in a qualitative fashion (e.g., interviews, analysis of QS meetup videos), but these patterns are difficult to probe in this way if we want an instantaneous characterization of commitment. Moreover, lack of standardization of use patterns in the aforementioned qualitative studies makes it difficult to draw meaningful comparisons among users. Use patterns have also been studied using more descriptive approaches, for example by inferring involvement through calculating activity logs. However, commitment does not follow automatically from demographic variables such as the kinds or number of trackers one owns, nor from descriptive statistics such as how frequently one tracks. These variables are expected to correlate to a certain extent; however, both ownership and frequency of use are deemed to be necessary but not sufficient indicators of a commitment to self-tracking. We argue that commitment characterizes a users' relationship with the technology. Hence, identifying and measuring behavioral facets of commitment enables us to unpack and understand the user-device relationship more systematically.

This paper makes two main contributions to the understanding of self-tracking: First, we add to the currently existing literature on the psychology of self-tracking by defining the construct "commitment to self-tracking," based on relevant behavioral manifestations as a proxy for assessing a user's commitment, and secondly, we have operationalized this construct by means of a self-report measure, the Commitment to Self-Tracking (C2ST) scale, which consists of 12 items, tapping in on those relevant behaviors. In what follows, we will describe the process of scale development and validation, and we will discuss scientific implications and potential practical applications of the C2ST scale.

\section{Method}

\subsection{Participants and design}

Participants were recruited via Amazon Mechanical Turk (MTurk). Sixty-two participants failed to pass the attention check questions. From the remaining sample, ten participants did not complete the questionnaire and were also excluded from further analyses. In total, the sample for the final analysis was composed of 300 participants (125 female), ranging in age from 18 to $69(M=32, S D=8.63)$. Recruited participants met the inclusion criteria of (1) owning a fitness tracker and (2) currently self-tracking at least one health-related lifestyle behavior (e.g., steps) using their self-tracker. Participants received 2 dollars as monetary compensation. The duration of self-tracker use prior to filling out the questionnaire varied from 1 to 84 months with a mean of 14 months and 47 days. The most frequently tracked feature was exercise; $88 \%$ of participants reported that they use their self-tracker to track their steps and workouts. Exercise is followed by sleep tracking and calorie tracking ( $51 \%$ and $47 \%$ of participants, respectively). More than half of the participants (56\%) reportedly checked the applications connected to their wearables on a daily basis to actively check their progress. While most of the participants were less likely to share their data with others (e.g., community, family, members), those who did share mostly preferred to do so on a weekly basis (14\%). 


\subsection{Item generation}

Prior to the current study, there was no systematic research investigating the construct of commitment in relation to selftracking behavior. This lack of exemplary work made it necessary to generate individual questionnaire items from scratch. We had two main sources of inspiration for item generation. First, we closely acquainted ourselves with the target group and their characteristics. This familiarization has taken place both in direct interaction with the target population (i.e., attending QS meet-ups) and through surveying interactions within the online self-tracking community (i.e., reviewing blogs and forums). In addition to this qualitative approach, we have also utilized existing literature for a review of complementary constructs of interests (e.g., motivation of selftracking).

\subsubsection{Measurement items generated from QS meet-ups, blogs, and forums}

A significant part of our inspiration has been derived inductively. QS meet-ups have provided opportunities for face-toface interactions with devoted self-tracker users, a particular group of people who are willing to allocate part of their leisure time to gather together, with the purpose of discussing their self-tracking experiences with each other. Authors of the present paper have attended QS Netherlands-Utrecht, QS Netherlands-Eindhoven, and QS Netherlands-Amsterdam meet-ups a number of times over the course of 2 years; content received through personal interactions and informal interviews with members of the QS community has contributed to the generation of items. During these meet-ups, individuals present their own self-experimentation projects and exchange experiences and insights with the audience. These videos of presentations are archived at QS blogs and are made accessible to the public. Studies by Choe et al. [5], Lee [21], Choe et al. [6], and Ruckenstein and Pantzar [34] have also addressed this particular source of content in their papers where extensive thematic analyses on the presentations were conducted. Taking these qualitative studies together with our own observations at QS meet-ups has helped identifying recurring patterns of QSers. Item 7 of the scale (I run analysis of my tracked behavior, see Appendix 1) is an example of this scrutinizing analysis; in many presentations, people were pointing out that they were taking extra steps to run a more tailored analysis for a better and enriched interpretation of the monitored data.

In addition to this, we performed extensive desk research on the various ways in which users of self-trackers express themselves in online platforms. These platforms, such as social media and forums, are forms of microblogging and constitute an important communication channel, allowing people to express their opinions in an unfiltered manner. Fitbit, as of today, is the biggest self-tracking wearable company on the market, making the Fitbit community one of the most actively used and frequently visited online forums. The questions posted in the forum vary considerably in topic ranging from asking for technical help (e.g., battery life) to exchanging motivating experiences (e.g., when did you realize that you were a Fitbit addict?). Given the main purpose of the study, these voluntarily shared first-hand user posts are worthwhile to delve into for a number of reasons. First of all, actively posting in forums in order to share one's own experience as well as to ask other fellow users' opinions requires dedication, at least to the extent that self-tracking has become a shareable part of one's everyday life. Moreover, exchanging experiences within the online community leaves the shared opinions intact from self-presentation concerns, at least to a certain degree, and thus leads to unprompted responses. Items 1, 2, 8, and 11 of the scale (When I forget my device at home, I would go back to get it; I sleep with my device on; I am willing to pay extra for a "good quality" tracker; I purchase accessories for my tracker) are examples of how we translated the input we have gained through such analyses into item generation. These items address users' dedication to their self-tracker and to what extent they are willing to invest in the device itself.

\subsubsection{Measurement items generated from the literature}

Throughout the item generation process, utilization of existing literature on relevant constructs of interest has accompanied our item generation approach. A better understanding of the systematic work done in motivation is essential as it is one of the most relevant complementary constructs to commitment. With regard to literature on motivation of self-tracking, the most important sources of inspiration included Gimpel et al. [13], Rooksby et al. [32], and Lupton [22]. By means of identifying underlying motives and drivers for self-tracking, these sources aided in categorization of user profiles and were helpful in translating these intentions (internal states) into their behavioral equivalents. The insights gained about intentions and motivations of self-tracking have contributed to formulating items that particularly address the functionality aspect of self-tracking. Items 3, 10, and 12 of the scale (I make extra effort to reach my goals; If someone beats me (in an online competition), I try to reconquer my position again; I update my pre-determined goals whenever required) are results of this process.

A user's commitment is expressed through the way that the data is interpreted and acted upon. We have also utilized literature on longitudinal studies in which participants are engaged with a self-tracking technology over a longer period of time and are asked to reflect upon their experiences the end of the study. Gouveia et al. [15], Fritz et al. [11], and Tang and Kay [40] are a few examples of these types of studies, 
contributing to our understanding of the extent to which longterm trackers differ in comparison with "newbies" in terms of their self-tracking routines. Additionally, in order not to limit our target group of interests to "extreme users," we have also studied literature that particularly addressed "ordinary" men and women quantifying their experiences, for example, Didžiokaitè et al. [8] and Pink et al. [28]. These studies provided depth needed for developing a scale that is applicable/ relatable for a wider range of self-tracker users. Such a comparison of literature about dedicated long-term users (e.g., members of the Quantified Self community) and unexperienced or non-expert trackers has revealed the difference in the role of self-tracking in their daily lives and the role of self-tracking in their social life. Items 4, 5, 6, and 9 of the scale (I share my data with others; I spread the idea of selftracking; I recommend self-tracking to others; I interact with a self-tracking community) emerged from this comparison. Responses given to these items enable us to differentiate between varying levels of one's socially projected self-tracker identity.

Please take note that the aforementioned sources are not the complete list of literature reviewed but they are only representative of their categories. Given that the main intention of the paper is scale development and not a systematic literature review, this listed literature serves to communicate the scope of themes that were reviewed during the item generation process. Overall, the C2ST scale is developed in such a way that its items altogether correspond to the aforementioned three defining elements of the construct of commitment to selftracking (i.e., self-tracker as device, self-tracking as functional activity seeking, and self-tracking as part of one's projected social identity). Based on the extensive desk research together with previously mentioned field research with Quantified Self meet-ups, a total set of 12 items was developed (see Appendix 1). Each item used a 7-point Likert type response scale, labeled from strongly disagree (1) to strongly agree (7).

\section{Results}

All analyses were performed with the Stata 16 statistical software package. To investigate the factorial structure behind our participants' responses to the 12 commitment items, we performed an exploratory common factor analysis (principal axis factoring). Due to the ordinal nature of the data, the analysis was performed on the polychoric correlation matrix. Factorability of the items was found to be good with sufficient inter-item correlations (all $r \mathrm{~s} \geq 0.32$ ) and excellent KaiserMeyer-Olkin (KMO) measures of sampling adequacy, with $\mathrm{KMO} \geq 0.89$ for individual items, and $\mathrm{KMO}=0.93$ overall. Kaiser's criterion revealed that one factor was to be retained with an eigenvalue of 6.5. Investigation of the scree plot and a parallel analysis $[14,9]$ suggested one, perhaps two factors to be retained. Therefore, we examined both a one- and a twofactor solution. For the latter, we used an oblique rotation using direct Oblimin. In the two-factor solution, 6 out of 12 items had low factor loadings (i.e., below $\lambda=0.60$ ) and there were multiple cross-loadings with $\lambda>0.30$. Moreover, we found the two extracted factors to be highly correlated with $r$ $=0.81$. Therefore, we decided against a two-factor solution. For the one-factor solution, factor loadings of all but one item exceeded $\lambda=0.60$ (see Table 1). Subsequently, we estimated factor scores by calculating, for each person, the average response across all 12 items (i.e., the summated scale method). The reliability of this measure (i.e., Cronbach's alpha) was high with $\alpha=0.91$.

\subsection{Validity assessment}

We expected that for a committed self-tracking user, the practice is freely endorsed and the underlying motivation is independent from externally referenced reasons such as rewards and punishments. Hence, the hypothesis was that commitment requires a higher degree of self-determination; those who engage in the practice to avoid negative consequences from external sources (e.g., disapproval of others) are less likely to manifest dedicated behavior. To test our hypothesis and to demonstrate convergent validity of the scale, the C2ST was evaluated through the pattern of correlations between the C2ST and a self-report motivation measure.

Self-determination theory (SDT) posits that there are different types of motivation that vary according to their level of self-determination. The taxonomy of motivation ranges from the most autonomous and highly self-determined forms (i.e., intrinsic motivation) to the most controlling and less selfdetermined forms of motivation (i.e., extrinsic motivation). Adjacent to intrinsic motivation on the continuum lies identified regulation, and it is followed by integrated regulation. They both encompass varying levels of autonomous

Table 1 Factor loadings $(\lambda)$ for each of the 12 items of the commitment scale

\begin{tabular}{ll}
\hline Item & Factor loading $(\lambda)$ \\
\hline CT1 & 0.64 \\
CT2 & 0.58 \\
CT3 & 0.62 \\
CT4 & 0.81 \\
CT5 & 0.82 \\
CT6 & 0.76 \\
CT7 & 0.70 \\
CT8 & 0.66 \\
CT9 & 0.82 \\
CT10 & 0.74 \\
CT11 & 0.72 \\
CT12 & 0.60 \\
\hline
\end{tabular}


motivation indicating that the engaged behavior is mostly selfdetermined. Adjacent to the extrinsic motivation on the continuum motivation lies introjected regulation, which holds a higher degree of controlled motivation where the quality of behavior is mostly non self-determined [35]. The Revised Sports Motivation Scale (SMS II) is a well-established measurement tool assessing sport motivation, and it adequately measures all types of motivation as covered by selfdetermination theory and thus serves our purpose of wellvalidated assessment of the motivational constructs of interest [26]. Few items of the SMS II were modified so that the items are fully applicable to the self-tracking context (e.g., original item because practicing sports reflects the essence of whom I am is modified to because self-tracking reflects the essence of whom I am). The original SMS II consisted of 18 items, with three items per type of motivation (intrinsic, identified, integrated, introjected, and external) and three items for amotivation. One item with the lowest alpha level for each motivation type was removed to decrease the length of the scale, while maintaining validity. Moreover, amotivation questions were inapplicable and thus left out as our targeted population consisted of people who are currently self-tracking, indicating that they likely had some level of motivation for wearing it. In the end, ten items remained for the motivation scale (see Appendix 2).

Since we were interested in the quality of motivation (presence vs. absence of self-determination) and not necessarily in the quantity of it (high vs. low), two subscales were calculated based on the degree to which the types of motivations are selfdetermined. Items measuring intrinsic, integrated, and identified motivation were grouped into a subscale measuring autonomous motivation [41]. Scoring high on autonomous motivation indicates that motivation is highly self-determined, reasons for self-tracking are self-directed, and that selftracking behavior is volitional and performed without pressure. Users practicing self-tracking for autonomous reasons are likely to engage in behaviors that are perceived to be consistent with their intrinsic goals and emanate from the self. Consisting of 6 items, autonomous motivation subscale had high reliability, Cronbach's $\alpha=0.79$. Similarly, items measuring introjected and extrinsic motivation types together formed a subscale measuring controlled motivation [41]. Scoring high on controlled motivation is interpreted as being likely to engage in behaviors due to reasons that are externally referenced such as avoiding feelings of guilt or gaining rewards. Users engaging in self-tracking practice for controlled reasons are likely to feel a sense of obligation and pressure when engaging in the behavior. Consisting of 4 items, controlled motivation also had good reliability, Cronbach's $\alpha=$ 0.78 .

We hypothesized that commitment correlates positively with autonomous motivation. In line with our expectations, results showed that commitment to self-track scores correlate significantly positively with autonomous motivation, $r=0.66$, $p<0.001$. Our findings also showed that commitment to selftrack correlates negatively with controlled motivation, $r=-$ $0.70, p<0.001$. Those who are highly committed to self-track were more likely to engage in self-tracking by autonomous reasons whereas the manifestation of commitment behavior decreases as the reasons for self-tracking shifts towards more controlled types of motivation. Overall, these findings support the convergent validity of the C2ST scale.

\section{Discussion}

Self-tracking technologies introduce novel everyday automation experiences which have the power to transform the ways in which users relate to their own bodies and behaviors. Monitored data have been found to influence the perception of one's own identity, up to a point where the archived data is perceived as a personal representation of the self [38]. Despite their increased and effortless integration into everyday life, to date, no tool had been developed to measure commitment to self-tracking in general. The work presented here is the first step towards conceptualization of commitment to selftracking and presents a tool for its measurement.

\subsection{Commitment to self-tracking as a new construct}

Commitment to self-tracking manifests itself in the way a user behaves in relation to self-tracking, in three distinct ways: the extent of a user's interaction with the self-tracking device itself; the level of personal investment in the self-tracking practice, be it through monetary investments, cognitive effort, or time; and finally, the extent to which self-tracking becomes a salient element in the individual's social identity. Given the increased importance of self-tracking in people's everyday lives, assessing users' commitment to self-tracking practice becomes a relevant measure for a better understanding of how self-tracking is being integrated and internalized by their users. To date, most academic work in the area of self-tracking has focused on a qualitative understanding of user motivations of self-tracking, as well as critical sociological and normative perspectives on the role of self-tracking in society. The psychological antecedents and consequences of self-tracking have received much less attention in scientific literature, and rigorous empirical studies into the motivational and behavioral effects of self-tracking have only recently emerged in literature. In order to improve our understanding of self-tracking practices, and the psychological consequences of self-tracking, we need to first have a firmer grasp of the level to which people are engaged with self-tracking. Such an understanding needs to go beyond the mere frequency with which selftrackers are being used at any one time, because, as we learned from previous literature, self-tracking behavior can be 
intrinsically and extrinsically triggered, and may occur for a wide variety of reasons, some of which are highly non-committal. Our study underscores this variation in commitment and proposes a new construct relevant to a more complete understanding of the psychology of self-tracking.

The work presented in this paper aims to make a first step towards a better understanding of the wide spectrum of behavioral manifestations related to self-tracking by introducing a new behavioral concept, commitment to self-tracking, and developing a scale for its measurement. The purpose of the present paper, then, was twofold: first, to introduce a conceptual definition of the construct of commitment to self-tracking, and second, to develop and validate a self-report scale that measures commitment to self-tracking, allowing an operationalization of the construct. Our study has led to the Commitment to Self-Tracking (C2ST) scale consisting of 12 self-report items. Factor analysis indicated that all the items fit onto a single theoretical construct, suggesting that the C2ST scale measures a unidimensional construct. This is somewhat at variance with how commitment is operationalized in organizational psychology, where it is treated more as a multidimensional construct (i.e., affective commitment, normative commitment, and continuance commitment; for a detailed explanation, see Allen and Meyer [1]). However, in the selftracking context, we explicitly conceptualize commitment as a unidimensional variable with underlying structures emerging from one single source consisting of users' actual behavior exhibited in relation to their self-tracking devices, contexts, and communities. Taken together, the scale's high inter-item reliability and high convergent validity with other conceptually related, but not identical, measures, bode well for the C2ST scale as a promising measure to assess commitment to self-tracking.

\subsection{The link between commitment and motivation in self-tracking}

In further analyses, we looked at the relationship between commitment and motivation and showed that commitment correlates positively with autonomous motivation and negatively with controlled motivation. These different forms of motivations reflect individuals' reasons and rationale for engaging in tasks. Autonomous motivation refers to being engaged in a behavior because it reflects personal interests and endorsement. Individuals with autonomous motivation are likely to persist with the engaged behavior with a sense of willingness and volition. Given this definition, it will come as no surprise that individuals who self-track with autonomous motivation exhibit more committed behavior towards self-tracking practice; those who enjoy self-tracking in and of itself, without the need for external reinforcements, seem to behave in a way that embraces this lifestyle. When behavior is determined by controlled motivation, on the other hand, one is likely to engage in a task because one feels compelled to do so by external (e.g., rewards) or internal (e.g., feelings of guilt or shame) pressures. Engaging in behaviors for controlled reasons tends to persist as long as these external reinforcements are present. Those who are self-tracking for controlled reasons seem to engage in the practice more out of a feeling of obligation and less because of their own volition, which forestalls expressing behaviors that are indicators of commitment. Having said this, there may be cases of controlled motivation (e.g., self-tracking as a prescribed medical regime) where some of the commitment-related behaviors may nevertheless be present (e.g., sleeping with the device on, or returning home to get the device when forgotten). To our knowledge, the participant sample in our study did not contain many such externally incentivized cases. This would be an interesting avenue of further investigation, as this may be a clear demonstration of the theoretical independence of motivational profile and commitment behavior. In general though, the negative correlation between commitment and controlled motivation is an intriguing finding as it provides experimental evidence for the inadequacy of conventional proxies for engagement, such as frequency of use.

\subsection{Future research}

Having said this, we acknowledge that a user's relationship with self-tracking is not static but it is very much dependent on technological as well as sociocultural developments. Since the C2ST scale is developed as a reflection of behavior, it is imperative to take technological developments into consideration as they have a direct impact on the way commitment behavior is manifested and the way everyday automation is experienced by the user. For example, we can imagine a future where nanotech self-tracking devices may be inserted under the skin. In fact, the use of microchips has already become a part of the self-tracking practice, albeit mostly among hobbyists who are tech-savvy enthusiasts with a keen interest in novel technological advancements [16]. Once such forms of self-tracking get adopted by a broader user base and become prevalent at a larger scale; the set of behaviors that manifest commitment to self-tracking may need to be revisited. For example, going back to fetch one's self-tracker if it was forgotten may not be such a relevant item for people carrying subcutaneous tracking devices. Additionally, although sociocultural changes take place in a rather slow motion in contrast to the rapidity of technological advancements, they are still important when assessing a user's relationship with selftracking practices. Integration of self-tracking technologies into our everyday life has been stimulating a particular understanding of our own health, where bodily routines are expected to conform to rigid norms (e.g., the norm that an average person should take 10,000 steps each day) [12]. This reshaping of health through techno-scientific developments 
has today established a ground for the rise of biomedicalization; health and corresponding constructs (i.e., taking care of chronic illnesses or following a healthy diet) have increasingly become encased in a discourse of numbers and values, symptoms and conditions, and diagnosis and treatment. All of this implies individual control and responsibility over one's health, and, importantly, individual culpability over one's ill health [25]. The reflection of this cultural change can be witnessed through the meaning ascribed to the selftracking practice. Today, having a salient self-quantifier identity is perceived as an indicator of being highly responsible for one's own health, which can increase the desire to manifest particular types of behaviors to strengthen this identity. Thus, taking sociocultural trends into consideration is important for redefining the role self-tracking technologies play in one's life and the social context in which interest and desire for selftracking is manifested.

By developing a scale that measures users' commitment to self-track, we aim to contribute to an understanding of the ways in which individuals interact with their technologies. Given the novel automation experiences self-tracking technologies offer in influencing how we connect with our own bodies, together with their ever-increasing usage by a range of different types of users, it is important to be able to make an objective comparison between users in terms of their commitment to self-tracking. The experiential effects stemming from self-quantification are rather unexplored, making it challenging to fully comprehend the long-term effects that this particular automation experience may have on a users' self-understanding. Take GPS trackers as an example, while incorporating such automation systems into our everyday life facilitates navigation, it, simultaneously, can erode our sense of direction simply because it is too convenient to offload the responsibility of guidance to the GPS itself [31]. Could it be that the use of self-tracking technologies also leads to similar experiences? Could the offloading of tasks to digital technologies alienate us from our bodies rather than facilitate the connection with our bodies? Having a measure that enables quantification of commitment to the practice of self-tracking is useful as well as practical in addressing such compelling questions.

\section{Conclusion}

The development of the current C2ST scale brings new opportunities to investigating automation experiences by making a useful first step in measuring commitment to self-tracking in a standardized and quantified way. It enables the systematic examination of bidirectional influences between commitment to self-track and automation experiences. Moreover, the C2ST scale allows investigating how commitment relates to other theoretical constructs relevant to automation experiences including self-tracking, motivation, and behavior change. To give an example, administration of the C2ST scale can provide insights regarding the abandonment problem which concerns the fact that the use of trackers usually drops off after 6 months, on average [19]. The role of commitment as a predictor of keeping users engaged over longer periods of time can and should be explored in future research. The scale also enables exploring how commitment changes across different user groups (e.g., male vs. female; young vs. old) and personality types, allowing for a more personalized approach in designing self-tracking technologies.

Overall, these results expand our understanding regarding the role of self-tracking in users' lives. Measurement of commitment using self-reported behaviors provides a novel and promising alternative for assessing users' varied relationships with self-tracking technologies. It brings a new holistic perspective in investigating everyday automation experiences in relation to self-tracking practices which had been quite limited in scope to date. By introducing the new construct of commitment to self-tracking and a tool for its' measurement, the current paper facilitates the further investigation of the relationship between commitment to self-tracking and everyday automation experiences both theoretically and empirically. The C2ST scale widens the scope of questions that can be asked in the personal informatics field and hence, correspondingly, creates a novel space for discussion on how the user connects to her body. Given the transforming role self-tracking technologies encompass in terms of connecting, understanding, and communicating with our bodies, the C2ST scale is helpful in assessing and better understanding the user and enables meaningful interpretation of users' experience with everyday automation.

While it is not without limitations, this current study presents a first attempt aiming at capturing commitment to selftracking. We believe that a reliable and valid measure of commitment to self-tracking can be helpful in enabling a better understanding of the variety of people's self-quantification behaviors, their psychological antecedents and consequences, and capturing users' interactions with these novel everyday automation experiences. This is of particular importance in a field that is moving from its qualitative and anecdotal beginnings towards a deeper and more systematic understanding of the underlying causal mechanisms based on rigorous psychological science.

Acknowledgments This work is part of the project "Mobile Support Systems for Behavior Change," supported by Netherlands Organization for Scientific Research (NWO). For more information see, http:// behaviour-change.eu/.

\section{Compliance with ethical standards}

Conflict of interest The author declare that they have no conflict of interest. 


\section{Appendix 1. Commitment to Self-Track (C2ST)} scale

1. If I forget my device at home, I would go back to get it.

2. I sleep with my device on.

3. I make extra effort to reach my goal

4. I share my data with others.

5. I spread the idea of self-tracking.

6. I recommend self-tracking to others.

7. I run an analysis of my tracked behavior (e.g., plot my running speed).

8. I am willing to pay extra for a "good quality" tracker.

9. I interact with a self-tracking community.

10. If someone beats me (in an online competition), I try to conquer my position again.

11. I purchase accessories for my tracker.

12. I update my pre-determined goals whenever required.

\section{Appendix 2. Motivation for self-tracking scale: adjusted from the Revised Sport Motivation Scale}

I self-track

1. Because it gives me pleasure to learn more about myself

2. Because it is very interesting to learn how I can improve myself

3. Because self-tracking reflects the essence of whom I am

4. Because self-tracking is an integral part of my life

5. Because I have chosen self-tracking as a way to develop myself

6. Because I found it is a good way to develop aspects of myself that I value

7. Because I would feel bad about myself if I did not take the time to do self-tracking

8. Because I feel better about myself when I do selftracking

9. Because people I care about would be upset with me if I didn't do self-tracking

10. Because I think others would disapprove of me if I didn't do self-tracking

Open Access This article is licensed under a Creative Commons Attribution 4.0 International License, which permits use, sharing, adaptation, distribution and reproduction in any medium or format, as long as you give appropriate credit to the original author(s) and the source, provide a link to the Creative Commons licence, and indicate if changes were made. The images or other third party material in this article are included in the article's Creative Commons licence, unless indicated otherwise in a credit line to the material. If material is not included in the article's Creative Commons licence and your intended use is not permitted by statutory regulation or exceeds the permitted use, you will need to obtain permission directly from the copyright holder. To view a copy of this licence, visit http://creativecommons.org/licenses/by/4.0/.

\section{References}

1. Allen NJ, Meyer JP (1990) The measurement and antecedents of affective, continuance and normative commitment to the organization. J Occup Psychol 63(1):1-18

2. Ajzen I, Fishbein M (1980) Understanding attitudes and predicting social behaviour. Prentice-Hall. Englewood Cliffs, New Jersey

3. Bode M, \& Kristensen DB (2015) The digital doppelgänger within: a study on self-tracking and the quantified self movement. In Assembling Consumption: Researching Actors, Networks and Markets. https://doi.org/10.4324/9781315743608

4. Bravata DM, Smith-Spangler C, Sundaram V, Gienger AL, Lin N, Lewis R, Stave CD, Olkin I, Sirard JR (2007) Using pedometers to increase physical activity and improve health: a systematic review. J Am Med Assoc 298(19):2296-2304. https://doi.org/10.1001/ jama.298.19.2296

5. Choe EK, Lee NB, Lee B, Pratt W, Kientz JA (2014) Understanding quantified-selfers' practices in collecting and exploring personal data. Conf Human Factors Comput Syst Proc: 1143, 1152. https://doi.org/10.1145/2556288.2557372

6. Choe EK, Lee B, Schraefel MC (2015) Characterizing visualization insights from quantified selfers' personal data presentations. IEEE Comput Graph Appl 35(4):28-37. https://doi.org/10.1109/MCG. 2015.51

7. Deci EL, Ryan RM (2008) Self-determination theory: a macrotheory of human motivation, development, and health. Can Psychol 49:182-185. https://doi.org/10.1037/a0012801

8. Didžiokaitè G, Saukko P, Greiffenhagen C (2018) The mundane experience of everyday calorie trackers: beyond the metaphor of Quantified Self. New Media Soc 20(4):1470-1487. https://doi. org $/ 10.1177 / 1461444817698478$

9. Dinno A (2009) Exploring the sensitivity of Horn's parallel analysis to the distributional form of random data. Multivariate Behavioral Research 44(3):362-388

10. Etkin $\mathrm{J}$ (2016) The hidden cost of personal quantification. J Consum Res 42(6):967-984. https://doi.org/10.1093/jcr/ucv095

11. Fritz T, Huang EM, Murphy GC, \& Zimmermann T (2014) Persuasive technology in the real world: a study of long-term use of activity sensing devices for fitness. Proceedings of the 32nd Annual ACM Conference on Human Factors in Computing Systems - CHI '14, 487-496. https://doi.org/10.1145/2556288. 2557383

12. Gilmore JN (2015) Everywear: The quantified self and wearable fitness technologies. New Media Soc 1984:1-16. https://doi.org/10. $1177 / 1461444815588768$

13. Gimpel H, Nißen M, Görlitz RA (2013) Quantifying the quantified self: a study on the motivation of patients to track their own health. Icis $2013 \mathrm{i}(3): 128-133$. https://doi.org/10.4156/jdcta.vol4.issue7.6

14. Glorfeld LW (1995) An improvement on Horn's parallel analysis methodology for selecting the correct number of factors to retain. Educ Psychol Meas 55(3):377-393

15. Gouveia R, Karapanos E, Hassenzahl M (2015) How do we engage with activity trackers? pp 1305-1316. https://doi.org/10.1145/ 2750858.2804290

16. Heffernan KJ, Vetere F, \& Chang S (2016) You put what, where? Hobbyist use of insertable devices. Conference on Human Factors in Computing Systems - Proceedings, May, 1798-1809. https://doi. org $/ 10.1145 / 2858036.2858392$

17. Kersten-van Dijk ET, IJsselsteijn WA (2016) Design beyond the numbers: sharing, comparing, storytelling and the need for a quantified us. Interact Des Archit 29(1):121-135

18. Kersten-van Dijk ET, Westerink JHDM, Beute F, IJsselsteijn WA (2017) Personal informatics, self-insight, and behavior change: a critical review of current literature. Hum Comput Interact 32(56):268-296. https://doi.org/10.1080/07370024.2016.1276456 
19. Kranz M, Möller A, Hammerla N, Diewald S, Plötz T, Olivier P, Roalter L (2013) The mobile fitness coach: towards individualized skill assessment using personalized mobile devices. Pervasive Mobile Comput 9(2):203-215. https://doi.org/10.1016/j.pmcj. 2012.06.002

20. Ledger D, McCaffrey D (2014) Inside Wearables: how the science of human behavior change offers the secret to long-term engagement. Retrieved from https://endeavourpartners.net/white-papers/. Accessed February 2020

21. Lee VR (2014) What's happening in the "quantified self" movement? Proceedings of International Conference of the Learning Sciences. ICLS 2(January):1032-1036 http://www.scopus.com/ inward/record.url?eid=2-s2.0-84937681741\&partnerID= 40\&md5=8f339111 ce28ed533db81b2e69038376

22. Lupton D (2014) Self-tracking modes: reflexive self-monitoring and data practices. SSRN Electronic Journal, October. https://doi. org $/ 10.2139 / \mathrm{ssrn} .2483549$

23. Lupton D (2018) 'I just want it to be done, done, done!' food tracking apps, affects, and agential capacities. Multimodal Technol Interact 2(2). https://doi.org/10.3390/mti2020029

24. Lyall B, Robards B (2018) Tool, toy and tutor: subjective experiences of digital self-tracking. J Sociol 54(1):108-124. https://doi. org $/ 10.1177 / 1440783317722854$

25. Neff G, Nafus D (2016) Self-Tracking. Cambridge, MA: MIT Press

26. Pelletier LG, Rocchi MA, Vallerand RJ, Deci EL, Ryan RM (2013) Validation of the revised sport motivation scale (SMS-II). Psychol Sport Exerc 14(3):329-341. https://doi.org/10.1016/j.psychsport. 2012.12.002

27. Perski O, Blandford A, Garnett C, Crane D, West R, Michie S (2019) A self-report measure of engagement with digital behavior change interventions (DBCIs): development and psychometric evaluation of the "DBCI Engagement Scale.". Transl Behav Med. https://doi.org/10.1093/tbm/ibz039

28. Pink S, Sumartojo S, Lupton D, Heyes La Bond C (2017) Mundane data: the routines, contingencies and accomplishments of digital living. Big Data Soc 4(1):205395171770092. https://doi.org/10. $1177 / 2053951717700924$

29. Piwek L, Ellis DA, Andrews S, Joinson A (2016) The rise of consumer health wearables: promises and barriers. PLoS Med 13(2):19. https://doi.org/10.1371/journal.pmed.1001953

30. Pourzanjani A, Quisel T, Foschini L (2016) Adherent use of digital health trackers is associated with weight loss. PLoS One 11: e0152504. https://doi.org/10.1371/journal.pone.0152504

31. Robbins J (2010) GPS navigation ... but what is it doing to us? IEEE Int Symp Technol Soc 2010:309-318. https://doi.org/10. 1109/ISTAS.2010.5514623

32. Rooksby J, Rost M, Morrison A, \& Chalmers M (2014) Personal tracking as lived informatics. Conference on Human Factors in Computing Systems - Proceedings, May, 1163-1172. https://doi. org/10.1145/2556288.2557039
33. Ruckenstein M (2014) Visualized and interacted life: personal analytics and engagements with data doubles. Societies 4(1):68-84. https://doi.org/10.3390/soc4010068

34. Ruckenstein M, Pantzar M (2017) Beyond the quantified self: Thematic exploration of a dataistic paradigm. New Media \& Society 19(3):401-418

35. Ryan R, Deci E (2000) Self-determination theory and the facilitation of intrinsic motivation, social development, and well-being. Am Psychol 55(1):68-78. https://doi.org/10.1037/0003-066X.55. 1.68

36. Sheeran P, Webb TL (2016) The intention-behavior gap. Soc Personal Psychol Compass 10(9):503-518. https://doi.org/10. $1111 /$ spc3.12265

37. Shih PC, Han K, Poole ES, Rosson MB, \& Carroll JM (2015) Use and adoption challenges of wearable activity trackers Erika Shehan Poole, The Pennsylvania State University Mary Beth Rosson, The Pennsylvania State University. IConference 2015: Create, Collaborate, Celebrate. (March 24-27, 2015), 1, 1-12.

38. Silverman J, Barasch A (2016) You are what you track: the effect of failing to log an experience on future use of tracking app. NA - Adv Consum Res 44:21-25

39. Simpson CC, Mazzeo SE (2017) Calorie counting and fitness tracking technology: associations with eating disorder symptomatology. Eat Behav 26(2017):89-92. https://doi.org/10.1016/j.eatbeh.2017. 02.002

40. Tang LM, \& Kay J (2017) Harnessing long term physical activity data-how long-term trackers use data and how an adherencebased interface supports new insights. Proceedings of the ACM on Interactive, Mobile, Wearable and Ubiquitous Technologies, 1(2), 1-28. https://doi.org/10.1145/3090091

41. Teixeira PJ, Carraça EV, Markland D, Silva MN, Ryan RM (2012) Exercise, physical activity, and self-determination theory: a systematic review. Int J Behav Nutr Phys Act 9(1):1. https://doi.org/10. 1186/1479-5868-9-78

42. Till C (2014) Exercise as labour: quantified self and the transformation of exercise into labour. Societies 4(SEPTEMBER 2014): 446-462. https://doi.org/10.3390/soc4030446

43. Viseu A, \& Suchman L (2010) Wearable augmentations. Technologized Images, Technologized Bodies: Anthropological Approaches to a New Politics of Vision, 161-184

44. Wiener Y, Gechman AS (1977) Commitment: a behavioral approach to job involvement. J Vocat Behav 10(1):47-52. https:// doi.org/10.1016/0001-8791(77)90041-0

45. Wolf G, (2008) WHY?. Quantified Self. https://quantifiedself.com/ blog/but-why/. Accessed 10 Feb 2020

46. Zuboff S (2019) The age of surveillance capitalism. Profile Books

Publisher's note Springer Nature remains neutral with regard to jurisdictional claims in published maps and institutional affiliations. 